\title{
Numerical analysis of shock wave and supersonic turbulent boundary interaction between adiabatic and cold walls
}

\section{Fulin Tong, Zhigong Tang, Changping Yu, Xingkun Zhu \& Xinliang Li}

To cite this article: Fulin Tong, Zhigong Tang, Changping Yu, Xingkun Zhu \& Xinliang Li (2017) Numerical analysis of shock wave and supersonic turbulent boundary interaction between adiabatic and cold walls, Journal of Turbulence, 18:6, 569-588, DOI: 10.1080/14685248.2017.1311017

To link to this article: http://dx.doi.org/10.1080/14685248.2017.1311017

曲 Published online: 07 Apr 2017.

Submit your article to this journal $₫$

Џ Article views: 39

Q View related articles $\square$

View Crossmark data ¿ 


\title{
Numerical analysis of shock wave and supersonic turbulent boundary interaction between adiabatic and cold walls
}

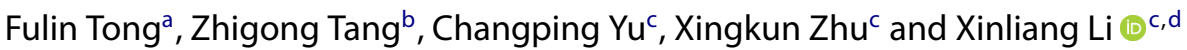 \\ ${ }^{a}$ China Aerodynamics Research and Development Center, Computational Aerodynamics Institute, Mianyang, \\ China; ${ }^{\mathrm{b} C h i n a}$ Aerodynamics Research and Development Center, Mianyang, China; ${ }^{\mathrm{C}} \mathrm{LHD}$, Institute of \\ Mechanics, Chinese Academy of Sciences, Beijing, China; ${ }^{\mathrm{d}}$ School of Engineering Science, University of \\ Chinese Academy of Sciences, Beijing, China
}

\begin{abstract}
Direct numerical simulations of shock wave and supersonic turbulent boundary layer interaction in a $24^{\circ}$ compression ramp with adiabatic and cold-wall temperatures are conducted. The wall temperature effects on turbulence structures and shock motions are investigated. The results are validated against previous experimental and numerical data. The effects of wall cooling on boundary layer characteristics are analysed. Statistical data show that wall cooling has a significant effect on the logarithmic region of mean velocity profile downstream the interaction region. Moreover, the influence of wall temperature on Reynolds stress anisotropy is mainly limited in the near-wall region and has little change on the outer layer. As the wall temperature decreases, the streamwise coherency of streaks increases. Based on the analysis of instantaneous Lamb vector divergence, the momentum transport between small-scale vortices on cold-wall condition is significantly enhanced. In addition, spectral analysis of wall pressure signals indicates that the location of peak of low-frequency energy shifts toward higher frequencies in cold case. Furthermore, the dynamic mode decomposition results reveal two characteristic modes, namely a low-frequency mode exhibiting the breathing motion of separation bubble and a high-frequency mode associated with the propagation of instability waves above separation bubble. The shape of dynamic modes is not sensitive to wall temperature.
\end{abstract}

\section{ARTICLE HISTORY}

Received 6 December 2016 Accepted 21 March 2017

\section{KEYWORDS}

Shock wave/turbulent boundary layer interaction; wall temperature; turbulence structure; shock motion; dynamic mode decomposition

\section{Introduction}

Shock wave and turbulent boundary layer interaction (SWTBLI) is an important fundamental aerodynamic problem, owing to its wide range of applications in the development of high-speed vehicles. The SWTBLI problem has been investigated extensively by experimental and numerical methods during the past 60 years.

Settles and Fitzpatrick [1] performed an experimental study to detail the interaction in compression ramp with increasing compression angle. The effects of shock strength on mean velocity profile and wall pressure were studied. Erengil and Dolling [2] investigated the unsteadiness in a Mach 5, $28^{\circ}$ separated compression ramp under approximately 
adiabatic wall conditions. The measured fluctuating wall pressure indicated that the unsteady motion of shock wave correlated with an expanding and contracting motion of bubble. Selig et al. [3] studied the change of turbulence structure through the interaction in compression ramp. A small correlation was found between turbulence amplification and unsteady shock motion. Above experiments were almost performed at relatively high Reynolds number. Recently, Bookey et al. [4] studied a compression ramp at Mach 2.9 and turning angle of $24^{\circ}$. This low Reynolds number experiment was within the reach of direct numerical simulation (DNS). Therefore, the obtained experimental database, including separation and reattachment points, mean wall pressure and velocity profiles, was used extensively to validate the DNS results.

Nowadays, DNS is playing a very important role in the studies of SWTBLIs. The first DNS of compression ramp was performed by Adam [5] at Mach $=3$ and $R e_{\theta}=1685$. The deflection angle was $18^{\circ}$. The origin of unsteady large-scale shock wave motion was analysed in detail using their DNS data. However, due to the limited Reynolds number, Adam's results cannot be validated by experiments. Wu and Martin [6] carried out a DNS of $24^{\circ}$ compression ramp at Mach 2.9 and $R e_{\theta}=2300$, matching the inflow conditions in experiment of Bookey et al. [4]. Their DNS results have shown good agreement with the experimental data. A feedback loop was proposed to explain the low-frequency (LF) unsteadiness of shock motion in their simulation [7]. Moreover, Martin et al. [8] studied the evolution of turbulence structure through compression ramp, including structure angle, streaks and hairpin packets. In addition, Ringuette et al. [9] used the same DNS data obtained by Wu and Martin [6] to analysis the effect of Reynolds number on wall pressure fluctuation and turbulence amplification.

Most previous studies of shock and turbulent boundary interaction have focused on adiabatic wall conditions. Actually, the change of wall temperature can have a significant impact on the interaction. Jaunet et al. [10] performed an experimental study of heated shock wave and boundary layer interaction, and the length and timescales of interaction were investigated. Recently, Zhu et al. [11] have studied the effect of wall temperature on the size of separation bubble in compression ramp and a semi-theoretical formula was proposed. Bernardini et al. [12] studied the heat transfer and wall temperature effects in SWTBLI. However, the research of wall temperature effect on the shock motion and turbulent fluctuations is still scarce.

In this paper, DNSs of shock wave and supersonic turbulent boundary layer interaction in a $24^{\circ}$ compression ramp with adiabatic and cold-wall temperature conditions are conducted to study the effect of wall temperature on the shock motions and turbulent structures. The adiabatic case is chosen to approach the experiment of Bookey et al. [4] and DNS of Wu et al. [6]. The obtained DNS results are validated against experimental measures and previous numerical data.

\section{Simulation details}

The compressible Navier-Stokes equations in curvilinear coordinates are employed:

$$
\frac{\partial U}{\partial t}+\frac{\partial\left(F_{c}+F_{v}\right)}{\partial \xi}+\frac{\partial\left(G_{c}+G_{v}\right)}{\partial \eta}+\frac{\partial\left(H_{c}+H_{v}\right)}{\partial \zeta}=0
$$


where $U$ denotes the conservative variables, $F_{c}, G_{c}$ and $H_{c}$ denote the convective terms in the $\xi, \eta$ and $\zeta$ directions. $F_{v}, G_{v}$ and $H_{v}$ are the corresponding viscous terms. The viscous stress and heat flux in viscous terms are obtained from the following Newtonian and Fourier models:

$$
\begin{aligned}
\sigma_{i j} & =\frac{2 \mu}{\operatorname{Re}}\left[\frac{1}{2}\left(\frac{\partial u_{i}}{\partial x_{j}}+\frac{\partial u_{j}}{\partial x_{i}}\right)-\frac{1}{3} \frac{\partial u_{k}}{\partial x_{k}} \delta_{i j}\right] \\
q_{j} & =-\frac{\mu}{(\gamma-1) \operatorname{Re} \operatorname{Pr} M_{\infty}^{2}} \frac{\partial T}{\partial x_{j}}
\end{aligned}
$$

where the perfect gas is selected as the working fluids. The temperature dependence of $\mu$ is based on Sutherland's law, and the thermodynamic variables are linked by the ideal gas law:

$$
\mu(T)=T^{3 / 2}(1+0.82) /(T+0.82), \quad p=\frac{\rho T}{\gamma M_{\infty}^{2}}
$$

An optimised weighted essentially non-oscillatory scheme proposed by Martin and Taylor [13] is used for the convective terms and an eighth-order central scheme for the viscous terms. Due to the symmetry stencils, the numerical dissipation of optimised scheme is very small, which is beneficial to resolve small-scale fluctuations. This scheme has been widely used in previous DNS of SWTBLI $[6,7,9,11]$. The temporal integration is conducted by using the explicit third-order Runge-Kutta method.

The free-stream Mach number $M_{\infty}$ is 2.9 and the free-stream temperature $T_{\infty}$ is $108.1 \mathrm{~K}$. Thus, the corresponding recovery temperature $T_{r}$ at wall is about $269.5 \mathrm{~K}$. In the present simulation, the wall temperature ratios $T_{w} / T_{r}$ for adiabatic case and cold case are 1.1 and 0.6 , respectively. Details of inflow free-stream and boundary layer parameters are both listed in Table 2.

The laminar profiles obtained from auxiliary simulation for flat-plate boundary layer under the same free-stream flow and wall temperature are used as inflow boundary conditions. Turbulence is triggered by the wall-normal blowing and suction perturbation proposed by Pirozzoli et al. [14]. In both cases, the parameters of perturbation, including streamwise location, amplitude and frequency, are the same with our previous work [15]. Periodic conditions are used in the spanwise direction. At the outlet and upper boundary, a non-reflecting boundary condition is used to avoid the reflection of small disturbances. In addition, a buffer region with progressively coarsening grid near the outlet is also used to further minimise the disturbances. Along the wall, the non-slip and isothermal wall conditions are applied.

Figure 1 shows the computational domain for both cases. The ramp corner is placed at $x=0$. The turning angle of compression ramp is $24^{\circ}$. The streamwise domain is partitioned into two zones: laminar-transition zone and interaction region. In the present simulations, the boundary layer thickness $\delta$ at reference station labelled in Figure 1 is used as the reference length. The reference station is selected at $x=-35 \mathrm{~mm}$, where the flow is fully developed turbulence in both cases. Details of domain size and grid resolution are reported in Table 1, where $L_{x}, L_{y}$ and $L_{z}$ indicate the length of domain in the streamwise, wall-normal and spanwise directions, respectively. $N_{x} \times N_{y} \times N_{z}$ represents the corresponding number 


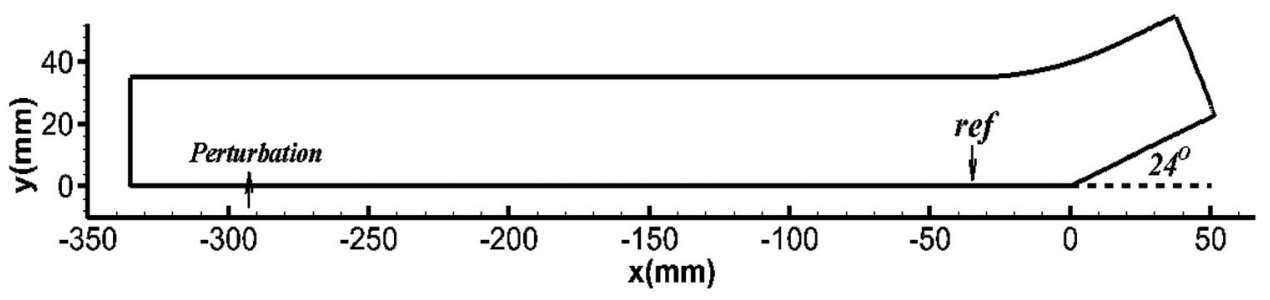

Figure 1. Computational domain.

Table 1. Domain size and grid resolution.

\begin{tabular}{lccccc}
\hline Wall condition & $N_{x} \times N_{y} \times N_{z}$ & $L_{x} \times L_{y} \times L_{z}(\mathrm{~mm})$ & $\Delta x^{+}$ & $\Delta y_{w}{ }^{+}$ & $\Delta z^{+}$ \\
\hline Adiabatic & $2160 \times 160 \times 140$ & $386.6 \times 35 \times 14$ & 3.74 & 0.43 & 4.28 \\
Cold & $3000 \times 200 \times 200$ & $386.6 \times 35 \times 14$ & 5.74 & 0.57 & 8.01 \\
\hline
\end{tabular}

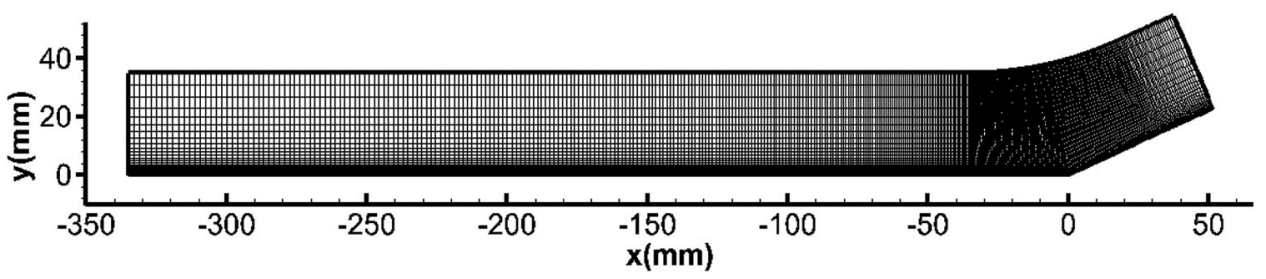

Figure 2. Computational mesh.

Table 2. Inflow free-stream and boundary layer parameters at the reference station.

\begin{tabular}{lcccccccc}
\hline & \multicolumn{3}{c}{ Inflow free-stream } & & \multicolumn{4}{c}{ Boundary layer parameter } \\
\cline { 2 - 4 } \cline { 6 - 9 } Wall condition & $M_{\infty}$ & $T_{\infty}(\mathrm{K})$ & $T_{w}(\mathrm{~K})$ & & $\Delta(\mathrm{mm})$ & $H$ & $R e_{\theta}$ & $C_{f}$ \\
\hline Adiabatic & 2.9 & 108.1 & 307 & & 6.5 & 5.02 & 2288 & 0.00256 \\
Cold & 2.9 & 108.1 & 102 & & 6.2 & 3.42 & 2623 & 0.00271 \\
\hline
\end{tabular}

of grid points. The computational mesh is sketched in Figure 2. For better visualisation, the mesh is plotted every five points in both streamwise and wall-normal direction. As shown in Figure 2, the grid points are concentrated in the interaction region $(-35 \mathrm{~mm} \leq x \leq$ $35 \mathrm{~mm}$ ) and near-wall region in the wall-normal direction. The grid points are equally spaced in the spanwise direction. In terms of wall units at reference station, the grid resolution in the interaction region of compression ramp for both cases is also given in Table 1.

Table 2 lists the boundary layer parameters at the reference station, where $\delta, H, \operatorname{Re}_{\theta}$ and $C_{f}$ denote the boundary layer thickness, the shape factor of boundary layer, Reynolds number based on the momentum thickness and skin-friction coefficient, respectively. It must be pointed out that the boundary layer thickness $\delta$ shown in this paper is taken at the reference station $(x=-35 \mathrm{~mm})$. According to the previous simulation [16], the matching Reynolds number $R e_{\theta}$ is used to keep the wall temperature as the only scaling parameter.

Figure $3(\mathrm{a}, \mathrm{b})$ plots the instantaneous temperature flow-fields in the middle section for cold case $\left(T_{w}=0.6 T_{r}\right)$ and the adiabatic case $\left(T_{w}=1.1 T_{\mathrm{r}}\right)$, respectively. As expected, transition to turbulence is triggered by the upstream disturbances. In both cases, the fully developed turbulent boundary layers are obtained. Note that the process of transition rapidly accelerates with the decreasing wall temperature [11]. In addition to, Figure 4 compared the mean skin-friction coefficient and theoretical estimates for the fully turbulent region 


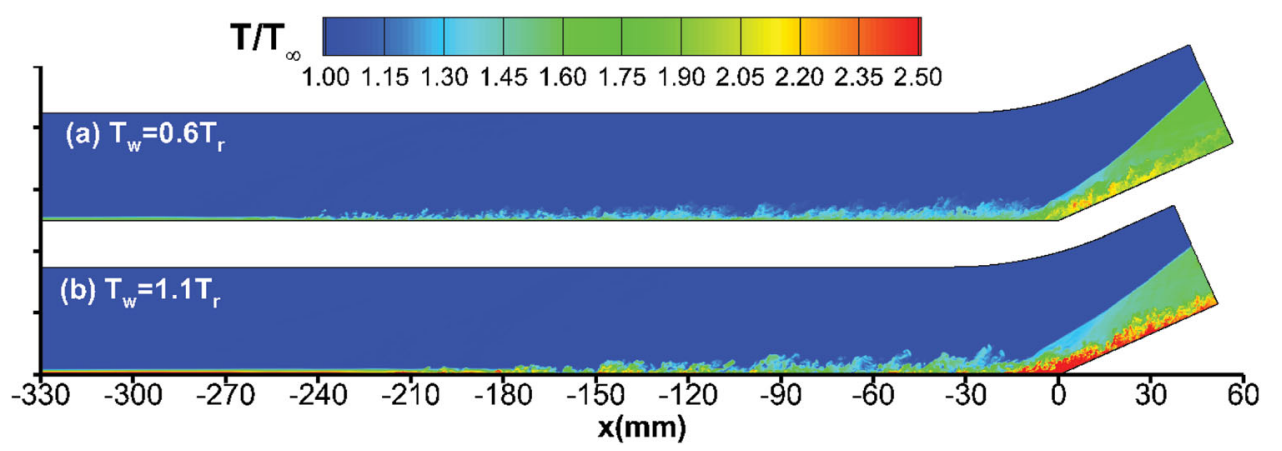

Figure 3. Instantaneous temperature in the middle section $(z=7 \mathrm{~mm}):(\mathrm{a}) T_{w}=0.6 T_{r^{\prime}}(\mathrm{b}) T_{w}=1.1 T_{r}$.

given by White [17]. It is shown that the simulation results agree well with the White correlation in the fully turbulent region, which also validates the method of transition triggered by blowing and suction.

After the flows reaches the statistically equilibrium state, the instantaneous flow-fields are collected every $1.23 \delta / U_{\infty}$, where $\delta$ is the boundary layer thickness at $x_{\text {ref }}$. The total number of flow-fields is 600 for cold case and 3000 for adiabatic case. To get the statistical data, time and spanwise average are used.

\section{Data validation}

To validate the grid resolution in terms of spanwise domain extent, the auto-correlation function in the spanwise direction at the reference location and $x / \delta=6$ is analysed in Figures 5 and 6 . These figures show that, whether at reference location or $x / \delta=6$, the correlations for velocity components for both cases decay towards zero after a distance of larger than half of the spanwise size of domain, which ensures that the computational domain

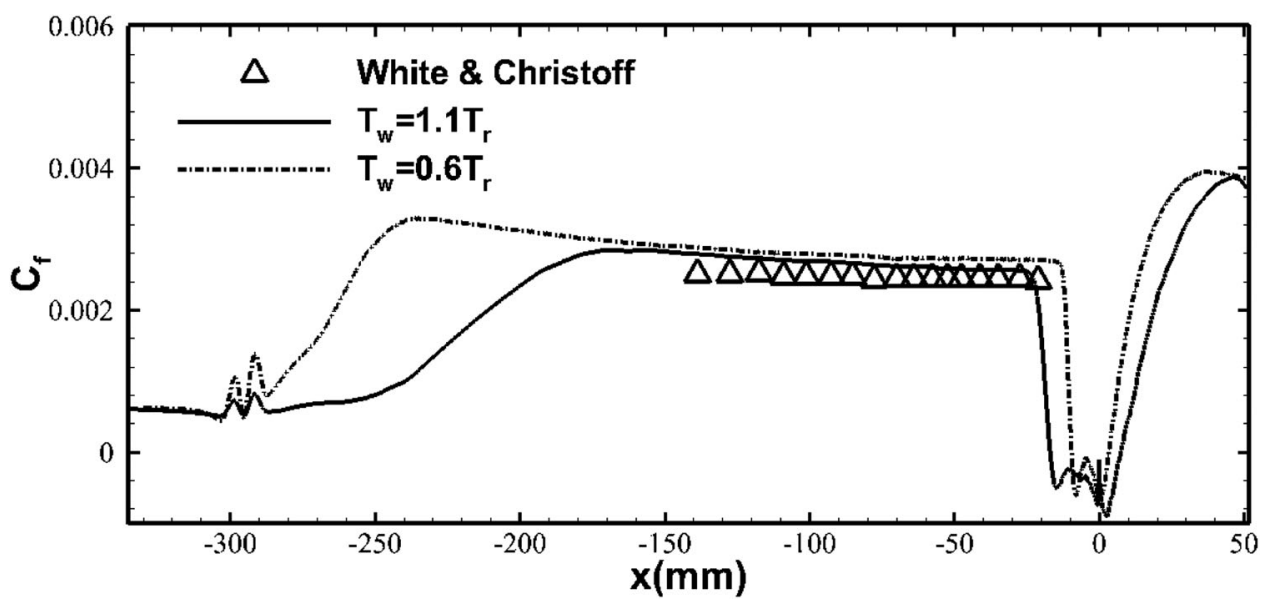

Figure 4. Streamwise distribution of wall mean skin-friction coefficient. 

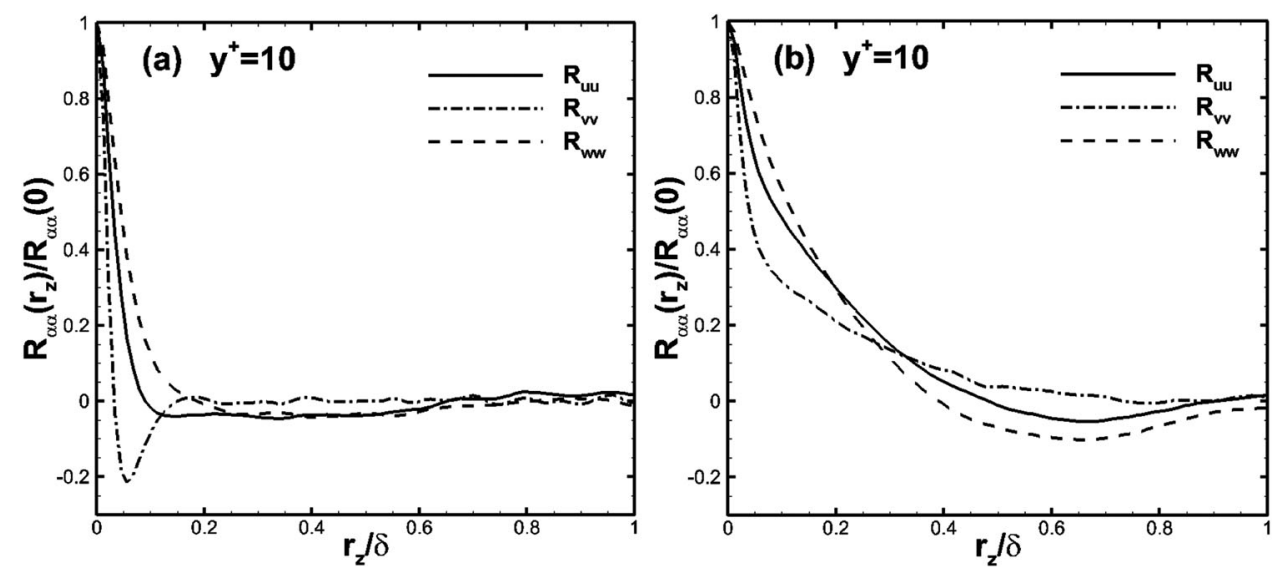

Figure 5. Two-point correlation at reference location (a) and $x / \delta=6$ (b) for cold cases.
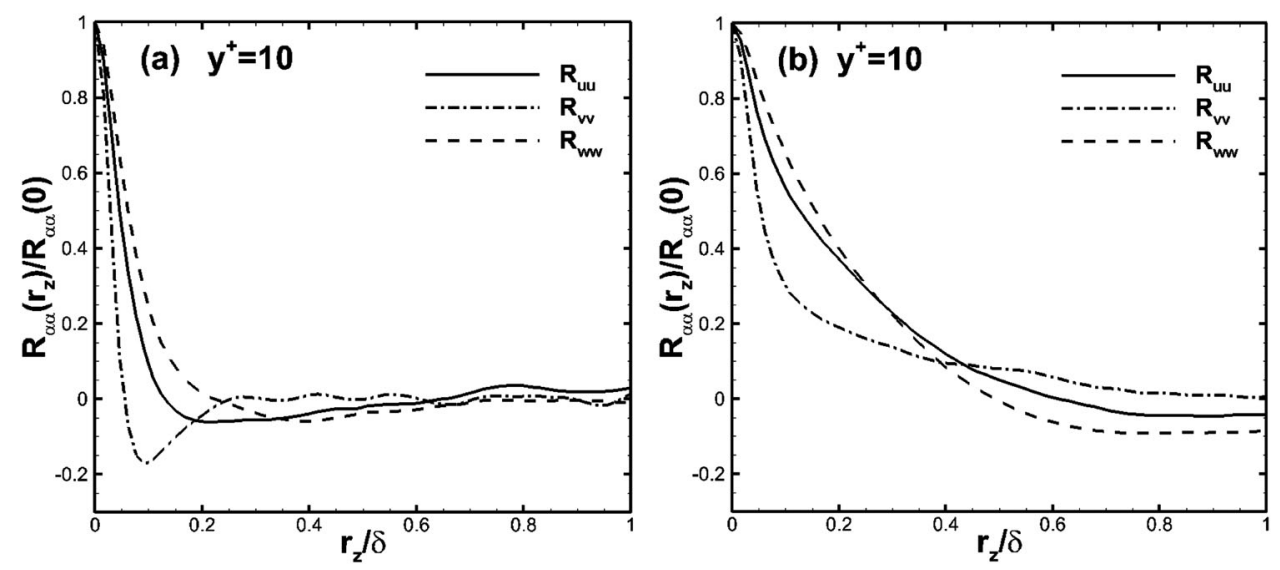

Figure 6. Two-point correlation at reference location (a) and $x / \delta=6$ (b) for adiabatic case.

in the spanwise direction is sufficiently wide to resolve the large-scale vortex structure in turbulence.

Figure 7 plots Van Direst transformed mean velocity profile at the reference station $(x=-35 \mathrm{~mm})$. It is shown that the profiles for adiabatic and cold-wall conditions agree well with the incompressible logarithm law in the logarithmic region. The profiles in the linear viscous sub-layer shrink with decreasing the wall temperature, which is also observed by previous findings [16]. Figure 8 plots the density-weighted turbulence intensities at reference station. The profiles collapse much better on the two wall temperature conditions. Moreover, as the density variation in boundary layer is taken into account, the results of present DNS match very well with that of incompressible DNS data [18] and low-speed boundary experiment [19].

To validate the accuracy of current DNS in the interaction region, Figure 9 plots the mean wall pressure distribution at adiabatic case. For comparison, the DNS results of Wu et al. [6] at $T_{w}=1.1 T_{r}$ have also been plotted and the black squares denote the experimental 


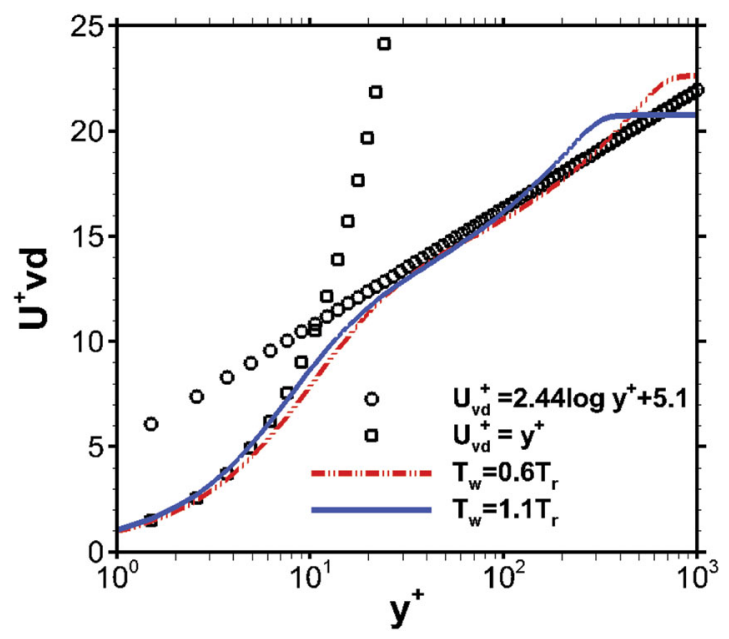

Figure 7. Van Driest transformed mean velocity profiles for different wall -temperature conditions at reference station.
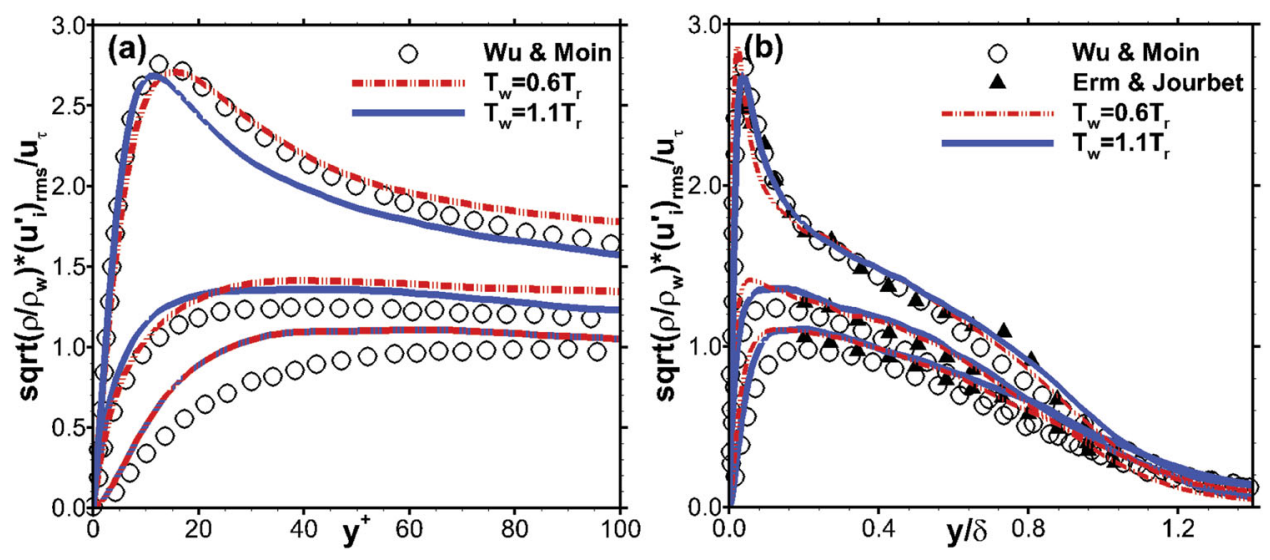

Figure 8. Density-weighted turbulence intensities for different wall temperature conditions at reference station: (a) inner scale; (b) outer scale.

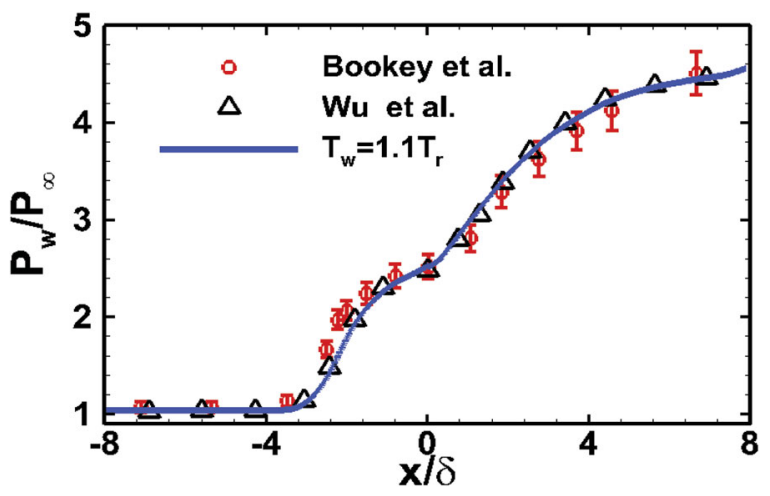

Figure 9. Streamwise distribution of the mean wall pressure in compression ramp. 

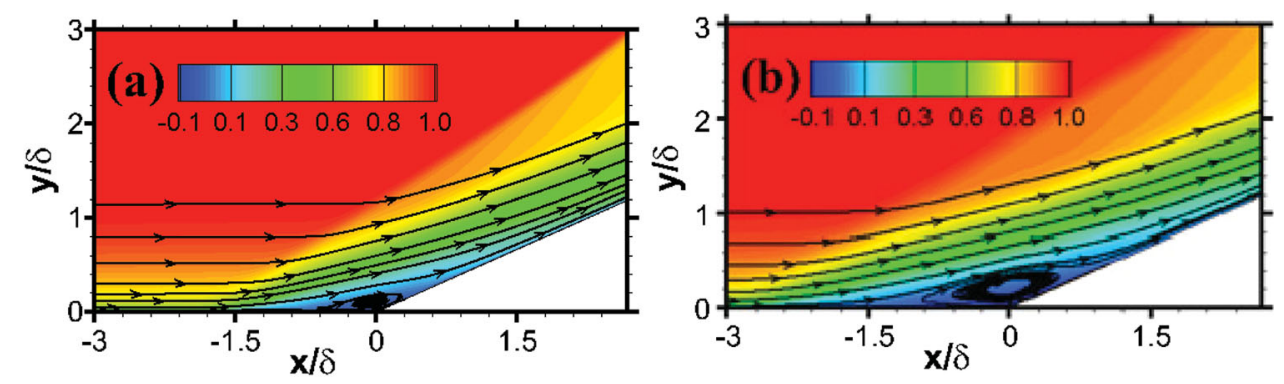

Figure 10. Mean streamwise velocity and streamlines: (a) $T_{w}=0.6 T_{r}$ (b) $T_{w}=1.1 T_{r}$.

data of Bookey et al. [4] with the error bar of 5\%. Evidently, the current simulation result is in good agreement with the DNS data of Wu et al. [6]. Furthermore, both DNS data predict the distribution of wall pressure within the experimental uncertainty. It is shown that there is a rapid pressure rise in the region $x / \delta \geq-3.5$, which is induced by the separation shock. Additionally, the increase of pressure, followed by a plateau value in the corner region, is presented at the reattachment region on ramp surface.

\section{Results and discussion}

\subsection{Flow visualisation}

The contours of mean streamwise velocity and streamlines are shown in Figure 10. The negative value of velocity indicates the existence of separation region in both cases. Obviously that as the wall temperature decreases, the separation region decreases. Figure 11 shows the streamwise distribution of wall skin-friction coefficient. This figure also shows that the length of separation region decreases significantly for the cold case. The length of separation bubble $\left(L_{\text {sep }}\right)$ decreases from $3.8 \delta$ for the adiabatic case to $2.0 \delta$ for the cold case. According to studies of Zhu et al. [11], the main reason to explain this reduction in separation region is a kind of Reynolds number effect, by the means of changing the density and viscosity in the near-wall region.

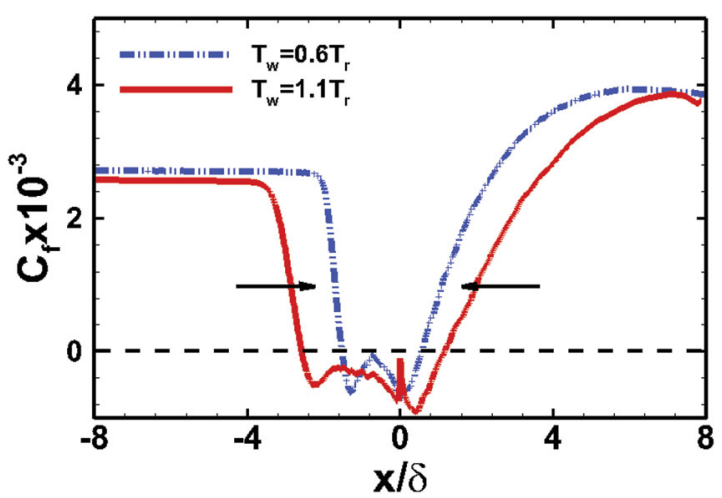

Figure 11. Streamwise distribution of the mean wall skin-friction coefficient in compression ramp. 

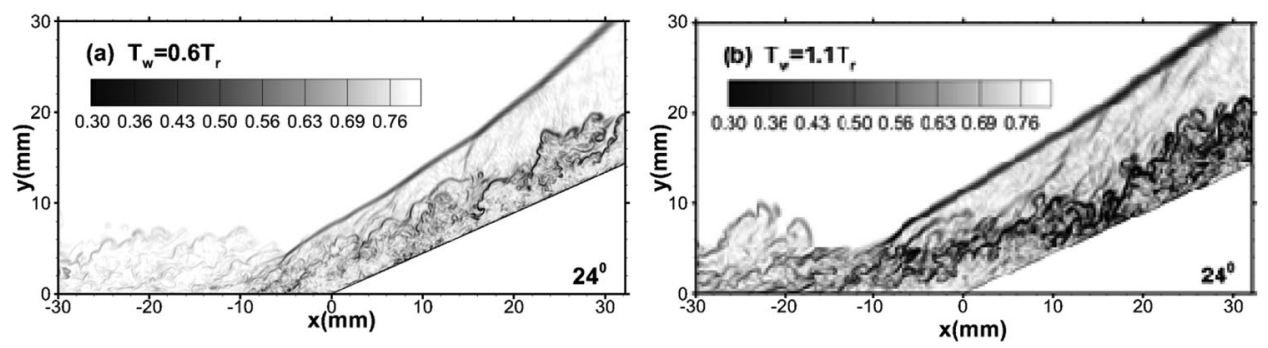

Figure 12. Instantaneous density gradient contours in the middle section.

Figure 12 plots the instantaneous density gradient contours for the adiabatic and cold case, respectively. The quantities are defined by Wu et al. [6]:

$$
N_{S}=0.8 \exp \left[-10\left(|\nabla \rho|-|\nabla \rho|_{\min }\right) /\left(|\nabla \rho|_{\max }-|\nabla \rho|_{\min }\right)\right]
$$

where $|\nabla \rho|$ is the density gradient. Regions of high density gradient in this figure are denoted with the dark regions. It is apparent that the intensity of turbulent fluctuation is dramatically amplified through the interaction with shock wave for the both cases. Whether the cold case or adiabatic condition, travelling shocklets extend out from the edge of the boundary layer downstream the interaction region. This figure shows that the main shock in cold-wall condition is much closer to the ramp surface, due to the smaller separation region. This figure also shows that the density gradient in the main shock region is stronger in the hotter wall case.

\subsection{Boundary layer evolution}

The development of mean velocity profile is shown in Figure 13(a,b) for various streamwise locations downstream of the reattachment point. We also can see the classical logarithmic law and linear relation in the same figure. Obviously, wall cooling has a significant
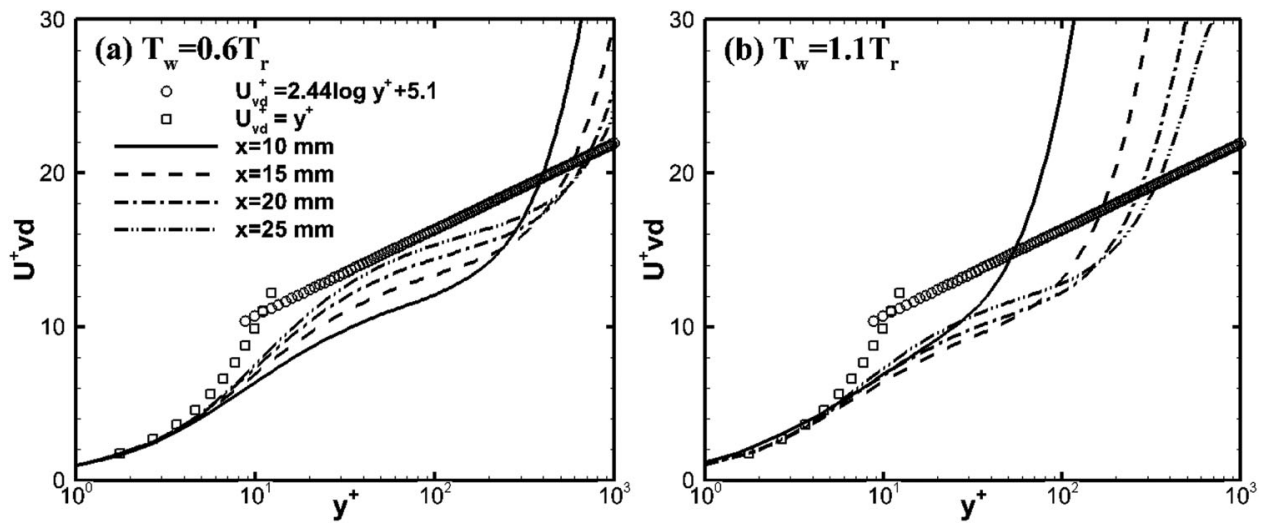

Figure 13. Van Driest transformed mean velocity profiles at various streamwise locations downstream of the reattachment point. 


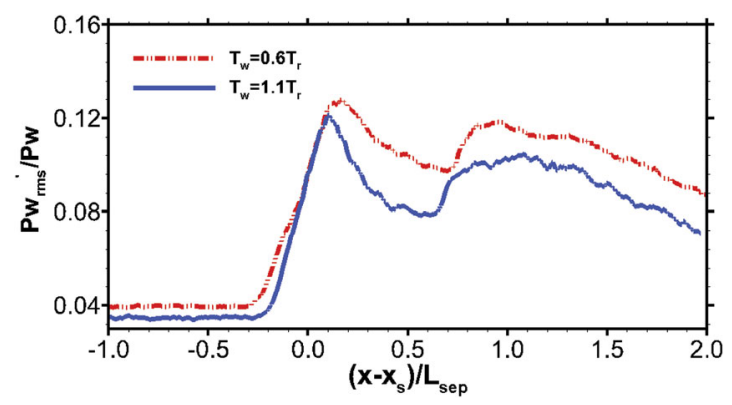

Figure 14. Streamwise distributions of wall pressure fluctuations normalised by the local mean wall pressure.

influence on the profile in the logarithmic region. For both temperature cases in this paper, the profiles downstream the interaction exhibit characteristic dips in the log-layer region. However, development into an equilibrium turbulent state is more rapid for the cold-wall case, especially in the log-layer region. From Figure 13(a) it can be seen that the velocity profile at $x=25 \mathrm{~mm}$ is close to the classical logarithmic law.

Figure 14 shows the streamwise variations of r.m.s. of wall pressure fluctuations, which are normalised by the local mean wall pressure and plotted against $x^{*}$. The variable $x^{*}$ is defined as $\left(x-x_{s}\right) / L_{\text {sep }}$, where $x_{s}$ is the mean separation point and $L_{\text {sep }}$ is the length of separation region. The data of adiabatic wall is consistent with the trend predicted by the data of cold wall. It is shown that there are two peaks in the intensity of pressure fluctuations, which present near separation and reattachment, respectively. Also note that the results of both temperature cases show a reasonable collapse in the regions upstream the separation.

The effect of wall temperature on Reynolds stress anisotropy is illustrated in Figure 15. This plot shows the second $\left(I I_{b}\right)$ and third $\left(I I_{b}\right)$ invariants of stress anisotropy tensor [20] for two wall temperature cases at selected streamwise stations. The invariants are defined as

$$
\begin{aligned}
b_{i j} & =\frac{<\rho u_{i}^{\prime \prime} u_{j}^{\prime \prime}>}{2<\rho><k>}-\frac{1}{3} \delta_{i j} \\
I I_{b} & =b_{i j} b_{j i} \\
I I I_{b} & =b_{i j} b_{j k} b_{k i}
\end{aligned}
$$

As previously shown by Lumley [20], all flow-fields obtained in current simulations lie inside the anisotropy invariant map. In the incoming boundary layer, Figure 15(a), as expected, a two component turbulence state is observed in the near-wall region and an isotropic state is gradually attained in the outer layer. The profiles are very similar to the numerical results of Pirozzoli [21] and Grilli [22]. The influence of wall cooling appears to become more pronounced in the near-wall region, where the maximum anisotropy is attained. Note that as the wall is cooled, the state moves much closer to one component turbulence. 

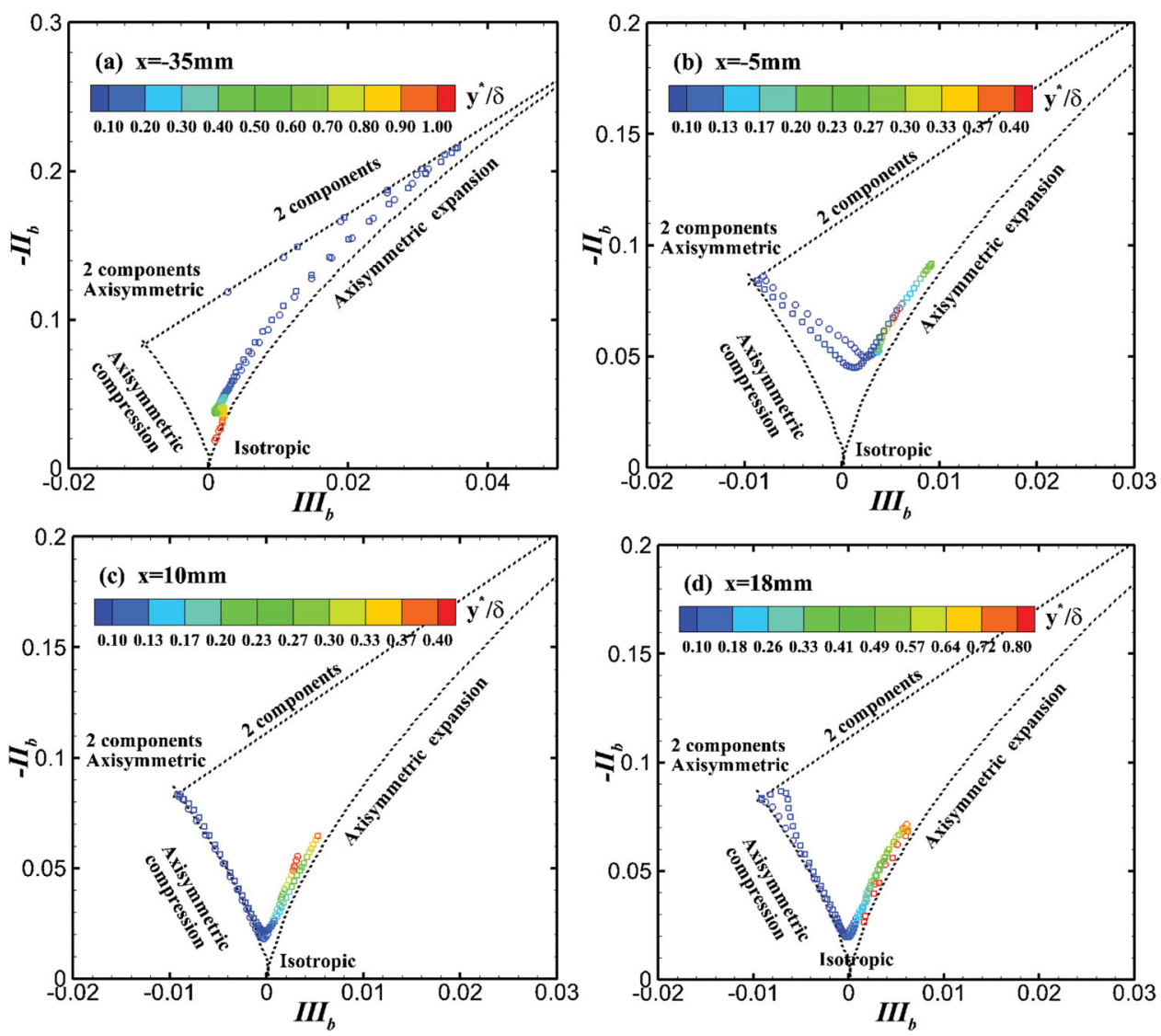

Figure 15. Reynolds stress anisotropy invariant map in the boundary layer at selected stations. The red lines denote cold case $\left(T_{w}=0.6 T_{r}\right)$; the circles denote adiabatic case $\left(T_{w}=1.1 T_{r}\right)$. The colour map indicates the normal distance from the wall.

Figure 15(b) shows the turbulent state in the separation region. The behaviour is totally different from that in the upstream region. It is shown that a two component axisymmetric turbulence is attained in the very near-wall region for both temperature cases. Moreover, in the inner boundary layer $\left(0.1<y^{*} / \delta<0.2\right)$, the anisotropy of turbulence is reduced by the existence of separation bubble and the turbulence resembles an isotropic state. It is interesting to note that the tendency to isotropic turbulence is stronger for the cold-wall case. However, the influence of wall cooling becomes much weaker in the outer region, where turbulence approaches an axisymmetric expansion state in the separated shear layer for both cases. The streamwise locations of plots in Figure 15(c,d) are downstream the reattachment point. It is found that the turbulence state close to the wall is characteristic of axisymmetric compression, which is associated with the adverse pressure gradient. Additionally, the results indicate that as the boundary layer develops downstream, the anisotropy in the near-wall region of cold-wall case exhibits an increasing tendency.

Figure 16 shows the instantaneous streamwise velocity field in a wall parallel plane at $y^{+}=5.5$ for adiabatic and cold-wall cases, respectively. The dash-dot lines denote the mean separation and reattachment points. This plot is set to highlight the change of 
(a)

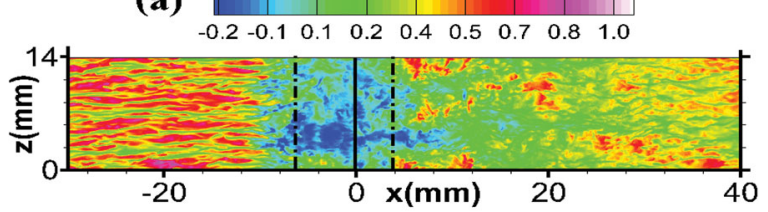

(b)

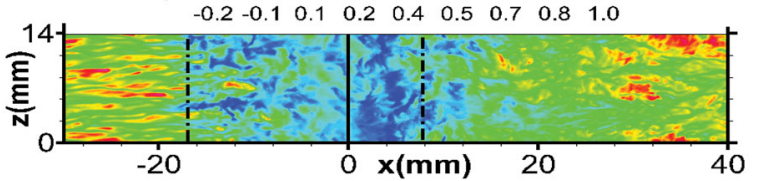

Figure 16. Distribution of the instantaneous streamwise velocity $\left(U_{s} / U_{\infty}\right)$ in a wall parallel pale at $y^{+}=$ 5.5: (a) $T_{w}=0.6 T_{r} ;$ (b) $T_{w}=1.1 T_{r}$.

turbulence structure through the interaction region. For the incoming boundary layer, a typical elongated streak structure is visible in both cases for $x<-20 \mathrm{~mm}$. Compared with the adiabatic case, spanwise meandering is decreased and streamwise coherency is increased for the cold case. This trend is also observed by the previous simulation [16,23]. In the separation region, denoted by blue colour map between the dash-dot lines, the streaky structures disappear and flow-field exhibits chaotic and isotropic. This scenario qualitatively confirmed the above analysis of Reynolds stress anisotropy in Figure 15(b). It is apparent that the tendency for streaks to recover in cold-wall case is much faster than the adiabatic case. There are two possible reasons accounting for the difference. First, the separation region is smaller and shock strength is weaker in the cold-wall case. Second, wall cooling has a significant reduction on the energy exchange in the near-wall region [16].

To characterise the momentum transport in compression ramp, Figure 17 plots the Lamb vector divergence [24], which is defined as

$$
\nabla \cdot L=\tilde{u} \cdot \nabla \times \omega-\tilde{\omega} \cdot \tilde{\omega}
$$
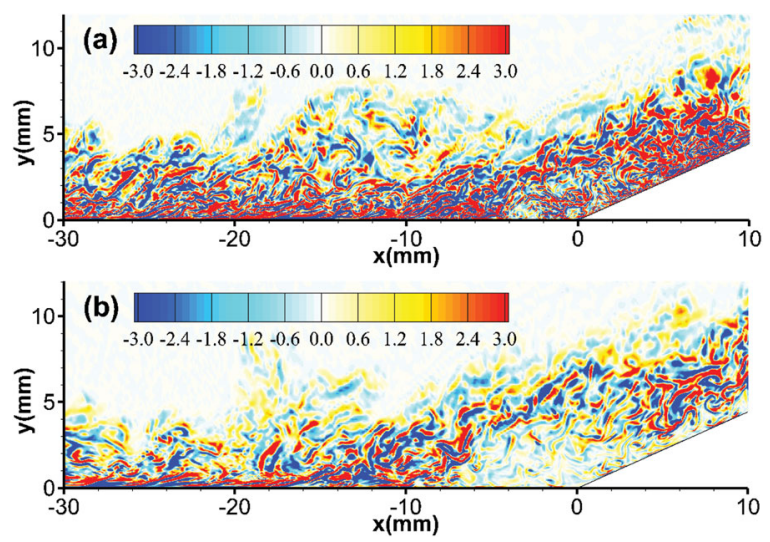

Figure 17. Distributions of the instantaneous Lamb vector divergence $(\nabla \cdot L)$ in the middle section: (a) $T_{w}=0.6 T_{r} ;$ (b) $T_{w}=1.1 T_{r}$. 


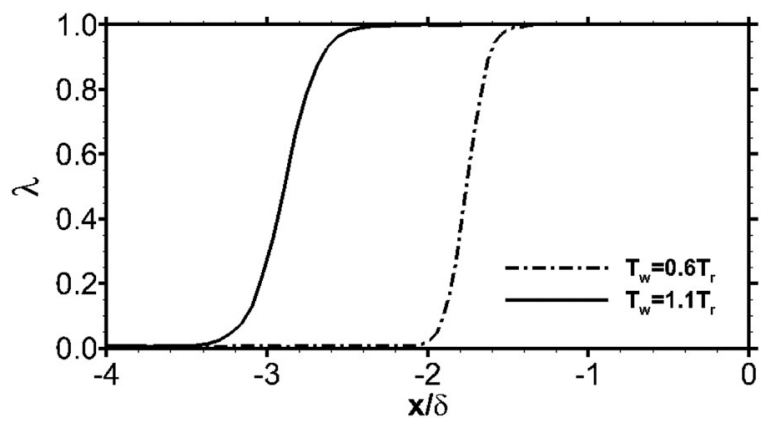

Figure 18. Intermittency function $(\lambda)$ computed from wall pressure signals.

where $\tilde{u} \cdot \nabla \times \omega$ denotes flexion product, $\tilde{\omega} \cdot \tilde{\omega}$ denotes the enstrophy. According to previous research [24], the negative value of Lamb vector divergence $\nabla \cdot L$ represents vorticity-bearing motions, while positive value is associated with straining motions. Figure 17 (a,b) shows the distribution of instantaneous Lamb vector divergence in the middle section for the adiabatic and cold case, respectively. It is clear that both the turbulent boundary layer and the separated shear layer are characterised by interval of negative and positive values with large magnitude, while in the shock wave region the Lamb vector divergence is relatively small. A similar trend is also observed from numerical investigation of the compressible flow past an aerofoil [25]. The major difference in the distribution of Lamb vector convergence for different wall temperature cases is presented in the near-wall region. Compared with the adiabatic results, the two-layer structures close to wall are more intensive and the scale is much smaller when the wall is cooled, as shown in Figure 17(a). This difference might be due to Reynolds number effect. When the wall temperature decreases, the mean density and viscosity near the wall change significantly. Therefore, the actual local Reynolds number defined by inflow parameters and wall values increases dramatically, which strengthens the momentum transport between small-scale vortices. Also note that the magnitude of Lamb vector divergence in the separation region is relatively small, which indicates a decrease of momentum transport.

\subsection{Shock wave motion}

The intermittency factor $\lambda$ is the fraction time that wall pressure exceeds a given threshold value, which is defined as

$$
\lambda=\frac{\operatorname{time}\left[P_{w}>\left(\left\langle P_{w I}\right\rangle+3 \sigma\left(P_{w I}\right)\right)\right]}{\text { totaltime }}
$$

where $\left\langle P_{w i}\right\rangle$ refers to the mean wall pressure at reference station and $\sigma\left(P_{w i}\right)$ represents the corresponding standard deviation. In order to estimate the length scale of shock unsteady motion, Figure 18 plots the intermittency $\lambda$ of wall pressure signals. The streamwise extent of shock motion can be estimated by the length over which $0.04 \leq \lambda \leq 0.98$. It is shown that the shape of profile is not affected by wall temperature. As wall temperature is decreased, the intermittency profile shifts towards ramp corner in the streamwise direction. The intermittent region is decreased from about $0.73 \delta$ for adiabatic case to $0.44 \delta$ for cold-wall case. 

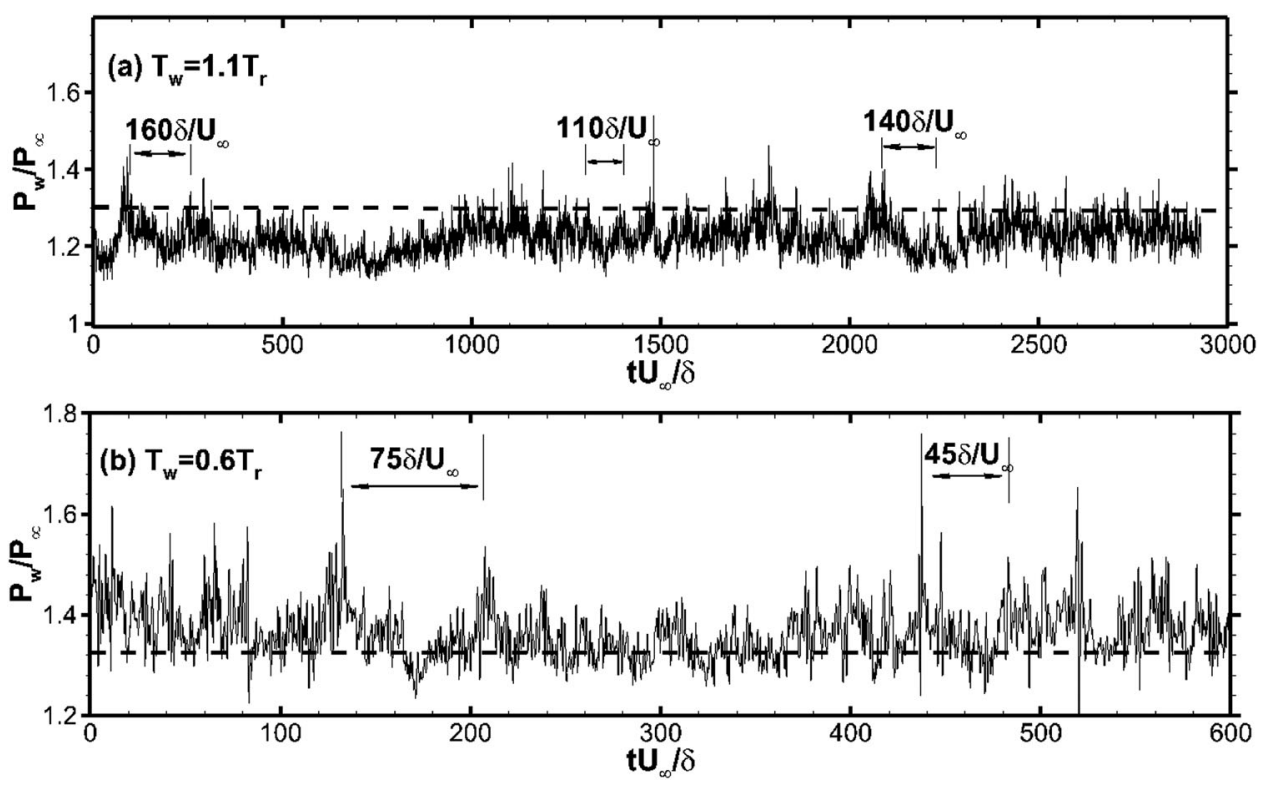

Figure 19. Wall pressure signal at mean separation point for adiabatic case (a) and cold-wall case (b). The dash line denotes time averaged wall pressure.

To further illustrate the effect of wall cooling on the LF unsteadiness of shock wave motion, Figure 19(a,b) shows the wall pressure signals at mean separation point for both cases. The signals are sampled in the middle section with $z=7 \mathrm{~mm}$. Time spans of the adiabatic and the cold case are 3000 and $600 \delta / U_{\infty}$, respectively. The sampling frequency is about $16 U_{\infty} / \delta$ for adiabatic case and $25 U_{\infty} / \delta$ for cold-wall case.

In Figure 19, a top-hat low-pass filter is applied to the raw signals to better display the LF unsteadiness. The cut-off frequency used in both cases is $U_{\infty} / \delta$, which is corresponding to the characteristic frequency in the incoming turbulent boundary layer. As shown in Figure 19(a), the motions over time scale of approximately $100 \delta / U_{\infty}$ are apparent. Three shock motions with time scale larger than $100 \delta / U_{\infty}$ are highlighted. According to the broadband character, it also exhibits the motions with shorter and longer time scales. When the wall is cooled, it is clear that the time scale of unsteady shock wave motion decreases to lower values, such as 75 and $45 \delta / U_{\infty}$ labelled in Figure 19(b), suggesting that the higher frequency are predominant in the cold wall than in the adiabatic case. This trend is consistent with the previous experimental results [10].

In addition, Figures 20 and 21 plot the streamwise evolution of weighted power spectral density (WPSD) as a function of non-dimensional frequency $\left(f \delta / U_{\infty}\right)$ for adiabatic and cold-wall cases, respectively. The quantity WPSD is defined as

$$
\operatorname{WPSD}(f)=f \cdot \operatorname{PSD}(f) / \int f \cdot \operatorname{PSD}(f) d f
$$

where PSD is the power spectral density of wall pressure signals. In the incoming turbulent boundary layer $(x / \delta=-5)$, as shown by Figures 20 and 21, the most energetic frequency 


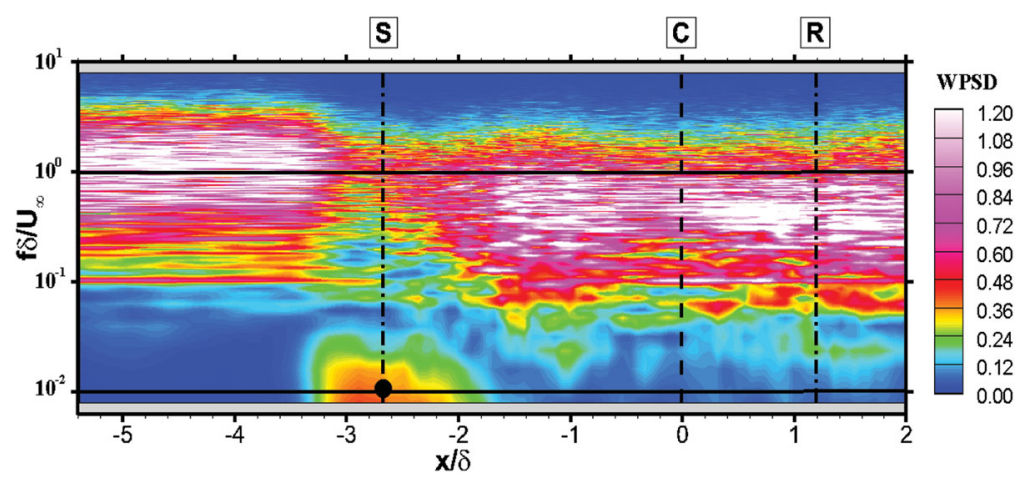

Figure 20. Weighted power spectral density (WPSD) of wall pressure signals for the adiabatic case.

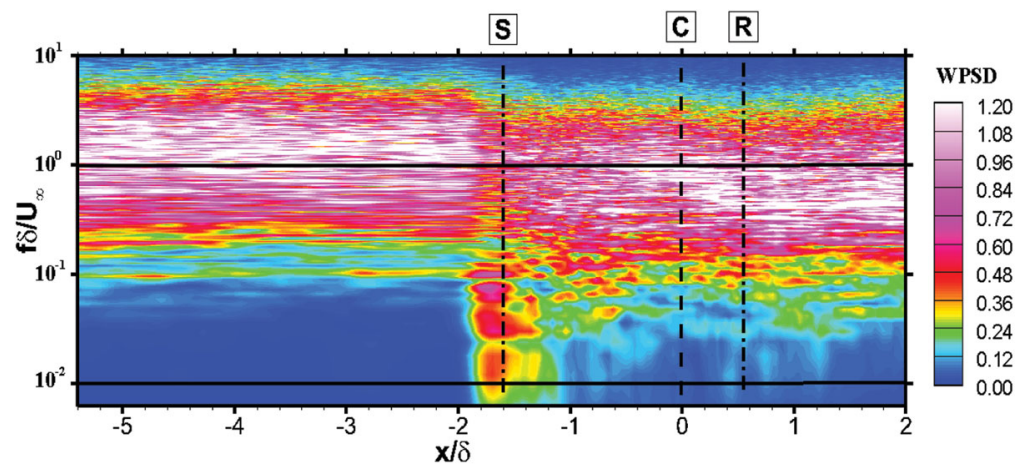

Figure 21. Weighted power spectral density (WPSD) of wall pressure signals for the cold case.

for both cases is about $U_{\infty} / \delta$. The effect of wall cooling on the high-frequency (HF) component is negligible. However, the spectrum of wall pressure signals at mean separation point (denoted by the symbol $S$ ) shows significantly wall temperature dependence. For the adiabatic wall condition, the peak of spectrum is centred on about $0.01 U_{\infty} / \delta$, which conforms to the previous findings of LF unsteadiness of shock motion $[6,22,26]$. In the study of shock and turbulent boundary interactions with adiabatic wall conditions, Dussauge [26] proposed a non-dimensional frequency that characterises the relationship between primary frequency of shock motion $(f)$ and length of separation bubble $\left(L_{\text {sep }}\right)$, which is defined as $\mathrm{St}=f L_{\mathrm{sep}} / U_{\infty}$. According to Figure 11, the separation region in adiabatic condition is about $3.8 \delta$. Therefore, the calculated Strouhal number (St) with adiabatic case in this paper is approximately 0.038 , consistent with previous numerical and experimental data $[6,26]$, namely $0.02-0.05$. However, as the wall is cooled, Figure 21 shows clearly that the most energetic frequency shifts toward higher frequency. Additionally, it is obvious that the spectrum in the cold interaction is characteristic of multi-peaks over $0.01-0.1 U_{\infty} / \delta$, which is also different from the adiabatic case.

\subsection{Dynamic mode decomposition}

Dynamic mode decomposition (DMD) method [27-29] is used to reveal the wall temperature effect on dynamical processes in shock wave and turbulent boundary interactions. 

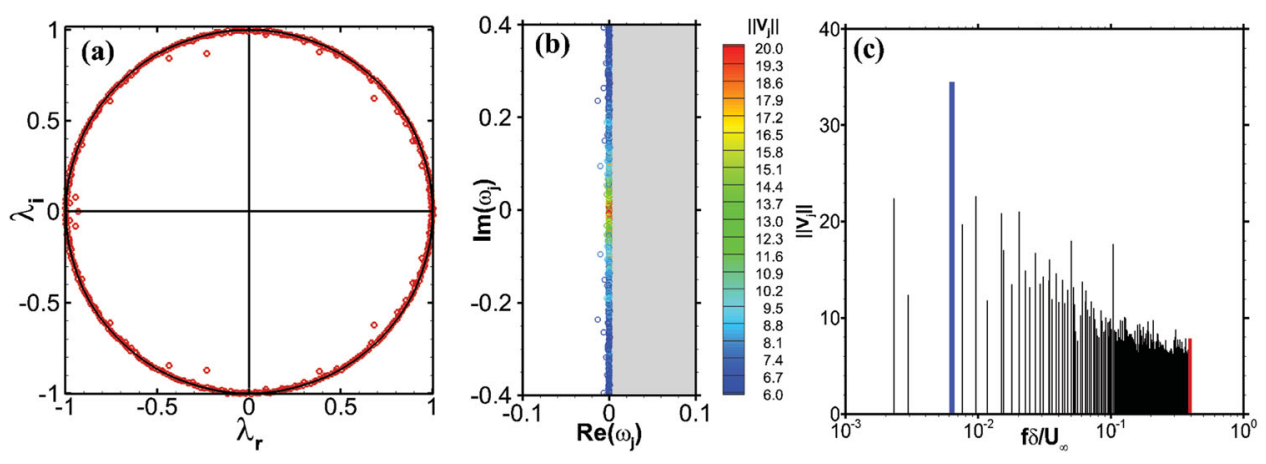

Figure 22. Spectrum of eigenvalues from the DMD of adiabatic wall case: (a) discrete spectrum; (b) continuous spectrum; (c) amplitude distribution.

The spanwise-averaged fluctuating streamwise velocity fields for both cases, between $-30 \mathrm{~mm}<x<20 \mathrm{~mm}$ in the streamwise direction and $0<y<10 \mathrm{~mm}$ in the wall-normal direction, are comparatively analysed. Based on the above analysis of flow-fields, the subdomain used in this paper is large enough to contain the shock wave and separation bubble. Snapshots are collected at constant time intervals of $0.81 \delta / U_{\infty}$ for cold case and $1.23 \delta / U_{\infty}$ for adiabatic case. The total number of snapshots are 640 and 400 , respectively. The corresponding range of resolvable frequency is $0.002-0.6 U_{\infty} / \delta$ and $0.002-0.4 U_{\infty} / \delta$.

Figures 22 and 23 plot the spectrum of complex eigenvalues detected by the DMD method for adiabatic case and cold case, respectively. Almost all eigenvalues fall on the unit circle, indicating the nonlinear system is statistically stationary. The continuous spectrum shaded by the amplitude of detected modes is present in Figures 22(b) and 23(b). Due to real-valued velocity fluctuations, the distribution of eigenvalues is symmetric. In addition, Figures 22(c) and 23(c) show the amplitude distribution of DMD modes as a function of non-dimensional frequency $f \delta / U_{\infty}$. Note that the peak value of modal amplitude appears in LF region for both wall conditions. Furthermore, the magnitude of LF modes is larger than that of HF modes.
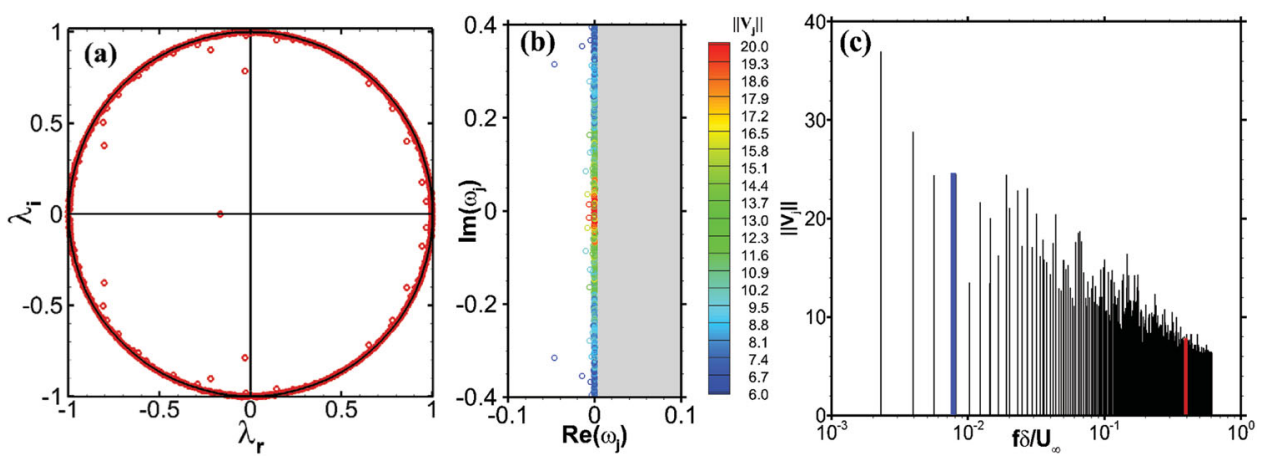

Figure 23. Spectrum of eigenvalues from the DMD of cold-wall case: (a) discrete spectrum; (b) continuous spectrum; (c) amplitude distribution. 

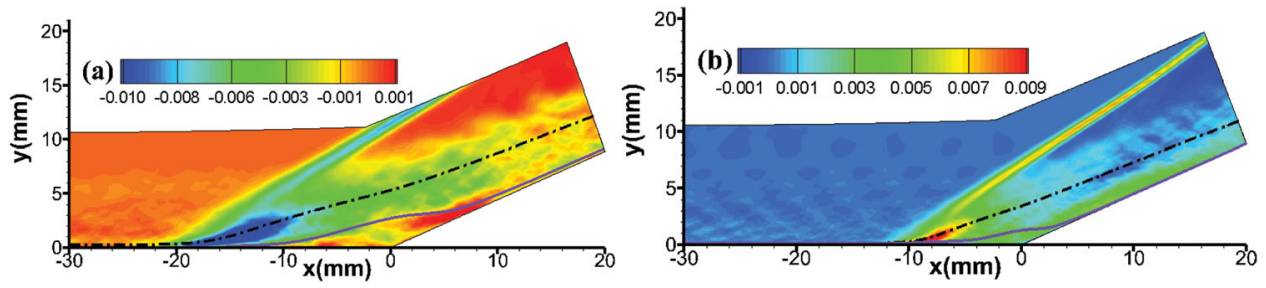

Figure 24. Visualisation of the real part of low-frequency mode indicated in Figures 22(c) and 23(c) by blue colour: (a) $T_{w}=1.1 T_{r}$ (b) $T_{w}=0.6 T_{r}$.

To further illustrate the coherent structure corresponding to characteristic frequency, the dynamic modes of LF and HF, labelled in Figures 18(c) and 19(c), are studied emphatically. The real parts of LF mode for adiabatic and cold-wall cases are shown in Figure 24. For comparison, the mean sonic line is indicated by the dash-dot line and the mean separating streamline is represented by the black solid line. The shape of LF mode is similar in both cases. A stronger fluctuation can be observed in the separated shear layer and shock wave region, particularly in the initial part of shear layer. However, the HF mode, as shown in Figure 25, exhibits a qualitative change. It is apparent that the fluctuation of HF mode is mainly concentrated around the mean sonic line. Furthermore, these fluctuations appear to travel downstream along the detaching shear layer above separation bubble. Also note that the wall cooling has little effect on the spatial organisation of the dynamic mode.

Figure 26 shows the dynamical process of LF mode for the adiabatic wall case. The flowfield corresponding to the characteristic frequency (labelled in Figure 22(c) by blue colour) is reconstructed and then added to the mean field. In Figure 26, the blue region in the ramp corner denotes separation bubble. It is found that the size of bubble starts to decrease, as shown in the first and second frame of Figure 26. Then, the bubble size increases again over the mean streamline in the fourth frame. The reconstructed flow-field is characteristic of contraction and expansion of bubble. The above modal analysis also suggests that the forcing mechanism of LF unsteadiness in shock and turbulent boundary layer interaction has strong relation with downstream-separated flows, rather than upstream turbulent structure.
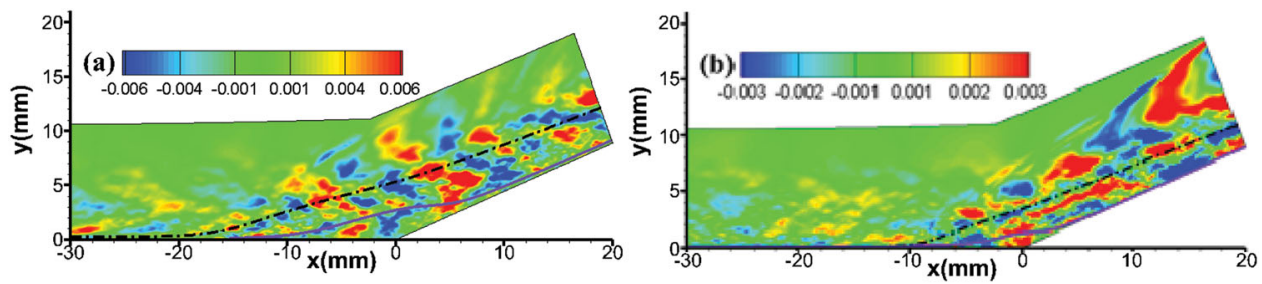

Figure 25. Visualisation of the real part of high-frequency mode indicated in Figures 23(c) and 24(c) by red colour: (a) $T_{w}=1.1 T_{r}$ (b) $T_{w}=0.6 T_{r}$. 

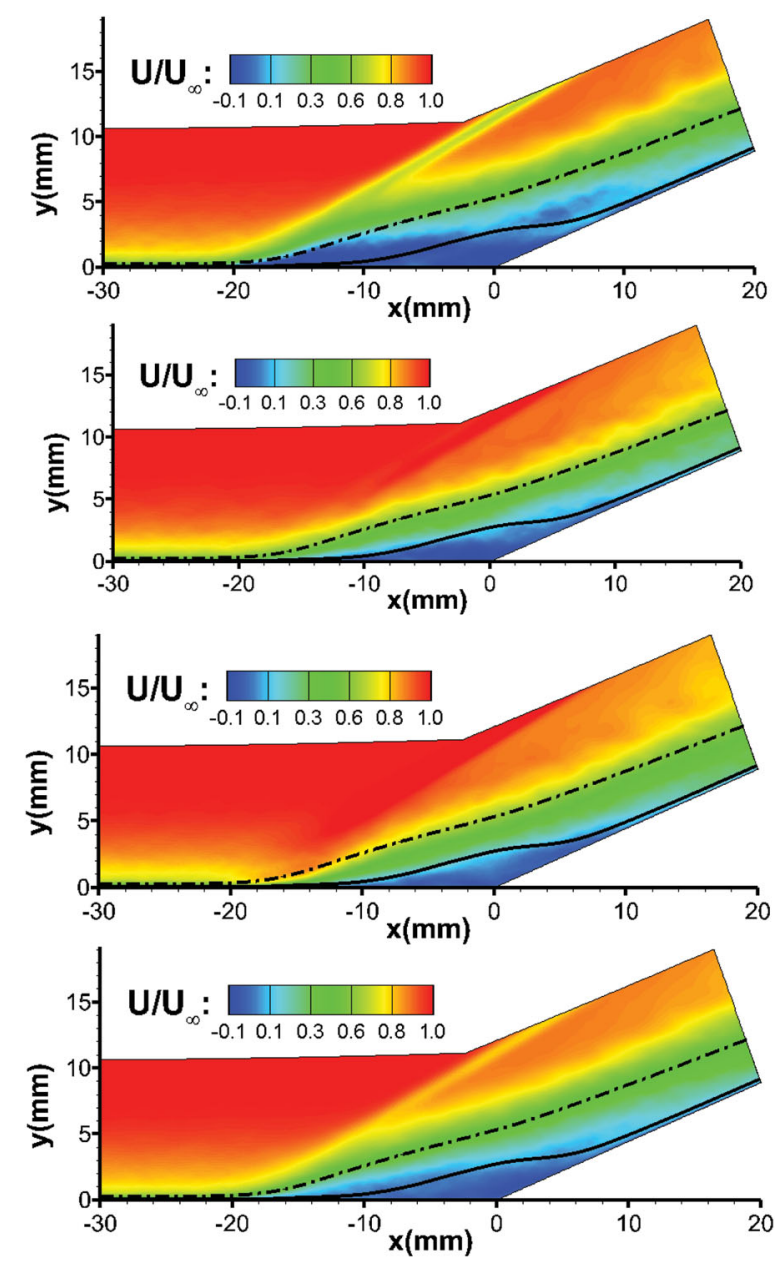

Figure 26. Four snapshots from reconstructed flow-fields of low-frequency mode for adiabatic wall case.

\section{Conclusion}

DNSs of a $24^{\circ}$ compression ramp with adiabatic and cold-wall condition are performed. The effect of wall temperature on shock wave and supersonic turbulent boundary layer interactions is studied. The accuracy of DNS results is validated against previous experimental and numerical data, including the upstream turbulent boundary layer, mean wall pressure and skin-friction distribution in the interaction region.

The boundary layer evolution throughout the interaction is analysed. Wall cooling has a significant effect on the development of mean velocity profile, particularly in the logarithmic region. Downstream the interaction, the flow recovery in cold case is much faster than the adiabatic case. The intensity of the fluctuating wall pressure for the two cases. It is found that the influence of wall temperature on Reynolds stress anisotropy is limited to near-wall region. Compared with the adiabatic wall case, the streamwise coherency of nearwall streaks in cold-wall case is increased. Analysis of instantaneous Lamb vector divergence indicates that the momentum transport in the interaction region is characteristic of 
two-layer structures with positive and negative value. Furthermore, the momentum transport between small-scale vortices on cold-wall condition is significantly enhanced due to Reynolds number effect.

The effect of wall temperature on length and time scales of shock motion is studied. When the wall temperature is decreased, the intermittent region of shock oscillation decreases and the location of peak LF energy shifts toward higher frequencies. The modal analysis of fluctuating streamwise velocity flow-field reveals that a LF mode exhibiting the breathing motion of separation bubble and a HF mode associated with the propagation of instability waves above separation bubble. In addition, the spatial organisation of obtained dynamic mode is not sensitive to wall temperature.

\section{Acknowledgments}

Thanks to Profs. Dexun Fu and Yanwen Ma in the Institute of mechanics, CAS, for their suggestions. This work is based on the research sponsored by the National Key research and Development Program of China (2016YFA0401200), the National Natural Science Foundation of China (Grant Nos. 91441103, 11372330 and 11472278) and Science Challenge Project (JCKY2016212A501). The authors would like to thank the National Computer Center in Tianjin (NSCC-TJ) and National Computer Center in GuangZhou (NSCC-GZ) and for providing computer time.

\section{Disclosure statement}

No potential conflict of interest was reported by the authors.

\section{Funding}

National Key Research and Development Program of China [grant number 2016YFA0401200]; National Natural Science Foundation of China [grant number 91441103], [grant number 11372330], [grant number 11472278]; Science Challenge Project [grant number JCKY2016212A501].

\section{ORCID}

Xinliang Li $₫$ http://orcid.org/0000-0002-4264-9620

\section{References}

[1] Settles GS, Fitzpatrick TJ. Detailed study of attached and separated compression corner flowfields in high Reynolds number supersonic flow. AIAA J. 1979;17(6):579-585.

[2] Erengil ME, Dolling DS. Unsteady wave structure near separation in a Mach 5 compression ramp interaction. AIAA J. 1991;29(5):728-735.

[3] Selig MS, Andreopoulos J, Muck KC, et al. Turbulence structure in a shock wave/turbulent boundary layer interaction. AIAA J. 1989;27(7):862-869.

[4] Bookey PB, Wyckham C, Smits AJ, et al. New experimental data of STBLI at DNS/LES accessible Reynolds numbers. In: 43th AIAA Aerospace Sciences Meeting and Exhibit; Reno, NV. AIAA Paper No. 2005-0309; 2005.

[5] Adams NA. Direct simulation of the turbulent boundary layer along a compression ramp at $M=3$ and $\operatorname{Re}_{\theta}=1685$. J Fluid Mech. 2000;420:47-83.

[6] Wu M, Martin MP. Direct numerical simulation of supersonic turbulent boundary layer over a compression ramp. AIAA J. 2007;45(4):879-889. 
[7] Wu M, Martin MP. Analysis of shock motion in shock wave and turbulent boundary layer interaction using direct numerical simulation data. J Fluid Mech. 2008;594:71-83

[8] Smits AJ, Martin MP, Wu M, et al. The turbulent structure of shock wave and boundary layer interaction in a compression corner. In: 44th AIAA Aerospace Sciences Meeting and Exhibit; Reno, NV. AIAA Paper No. 2006-0497; 2006.

[9] Ringuette MJ, Wu M, Martin MP. Low Reynolds number effects in a Mach 3 shock turbulent boundary layer interaction. AIAA J. 2008;46(7):1884-1887.

[10] Jaunet V, Debieve JF, Dupont P. Length scales and time scales of a heated shock wave/boundary layer interaction. AIAA J. 2014;52(11):2524-2532.

[11] Zhu XK, Yu CP, Tong FL, et al. Numerical study on wall temperature effects on shock wave/turbulent boundary-layer interaction. AIAA J. 2017;55(1):131-140.

[12] Bernardini M, Asproulias I, Larsson J, et al. Heat transfer and wall temperature effects in shock wave turbulent boundary layer interactions. Phys Rev Fluids. 2016;1:084403.

[13] Martin MP, Taylor EM, Wu M, et al. A bandwidth-optimized WENO scheme for the effective direct numerical simulation of compressible turbulence. J Comput Phys. 2006;220: 270-289.

[14] Pirozzoli S, Grasso F, Gatski TB. Direct numerical simulation and analysis of a spatially evolving supersonic turbulent boundary layer at $\mathrm{M}=2.25$. Phys Fluids. 2004;16:530-545.

[15] Li XL, Fu DX, Ma YW. Direct numerical simulation of shock/turbulent boundary layer interaction in a supersonic compression ramp. Sci Chin Phys Mech Astron. 2010;53(9): 1651-1658.

[16] Duan L, Beekman I, Martin MP. Direct numerical simulation of hypersonic turbulent boundary layers. Part 2: effect of wall temperature. J Fluid Mech. 2010;655:419-445.

[17] White FM, Corfield I. Viscous fluid flow. New York (NY): McGraw-Hill; 2006.

[18] Wu X, Moin P. Direct numerical simulation of turbulence in a nominally zero-pressuregradient flat-plate boundary layer. J Fluid Mech. 2009;630:5-41.

[19] Erm LP, Joubert J. Low Reynolds number turbulent boundary layers. J Fluid Mech. 1991;230: $1-44$.

[20] Lumley JL. Computational modeling of turbulent flows. Adv Appl Mech. 1978;18:123-176.

[21] Pirozzoli S, Bernardini M, Grasso F. Direct numerical simulation of transonic shock/boundary layer interaction under conditions of incipient separation. J Fluid Mech. 2010;657:361-393.

[22] Grilli M, Hickel S, Adams NA. Large-eddy simulation of a supersonic turbulent boundary layer over a compression-expansion ramp. Int J Heat Fluid Flow. 2013;42:79-93.

[23] Coleman GN, Kim J, Moser RD. A numerical study of turbulent supersonic isothermal-wall channel flow. J Fluid Mech. 1995;305:159-183.

[24] Hamman CW, Klewicki JC, Kirby RM. On the Lamb vector divergence in Navier-Stokes flows. J Fluid Mech. 2008;610:261-284.

[25] Chen LW, Xu CY, Lu XY. Numerical investigation of the compressible flow past an aerofoil. J Fluid Mech. 2010;643:97-126.

[26] Dussauge JP, Dupont P, Debieve JF. Unsteadiness in shock wave boundary layer interactions with separation. Aerosp Sci Technol. 2006;10(2):85-91.

[27] Schmid PJ. Dynamic mode decomposition of numerical and experimental data. J Fluid Mech. 2010;656:5-28.

[28] Grilli M, Schmid PJ, Hickel S, et al. Analysis of unsteady behavior in shock wave turbulent boundary layer interaction. J Fluid Mech. 2012;700:16-28.

[29] Pirozzoli S, Larsson J, Nichols JW, et al. Analysis of unsteady effects in shock/boundary layer interactions. Proceedings of the Summer Program 2010; Center for Turbulence Research, Stanford University; 2010; p. 153-164. 